\title{
THE INTEGRAL ABILITIES OF THE PERSONALITY AS THE SUBJECT MATTER OF PSYCHOLOGICAL RESEARCH
}

\author{
Anatoly V. Karpov \\ P. G. Demidov Yaroslavl State University \\ Yaroslavl, Russia
}

This article discusses some key methodological and theoretical issues of the fundamental, common psychological problem of abilities. It shows that there is a lack of modern ideas about the content of the subject matter of the psychology of abilities and, therefore, a need for enrichment. In this conceptual expansion it is necessary to differentiate a special, qualitatively specific class of abilities - the integral abilities of the individual. All these abilities are differentiated on the basis of the most promising paradigm in the development of the ability problem- the functional-genetic paradigm - and are related to one of the basic classes of mental processes - the integral mental processes (goal formation, anticipation, decision making, programming, prognosis, planning, control, self-control). In the article a detailed psychological characteristic of the basic integral abilities is presented, as a result of which the correspondence of these abilities to all features and criteria of abilities as such is proved. The necessity of using the structural-level approach in relation to the ability problem is shown, and a holistic, generalized hierarchy of the basic ability classes is proposed. This hierarchy has three main levels. At the highest level the general abilities are located; at the basic level are the specific abilities of a person. The integral abilities are located between these two levels and thus form a mesolevel in the general hierarchical organization of the abilities of the individual.

Keywords: ability, specific abilities, general abilities, integral abilities, functionalgenetic paradigm, structural-level approach, the hierarchy of abilities, goal formation, anticipation, decision making, prognosis, programming, planning, control, self-control.

\section{I}

The problem of abilities is considered one of the most fundamental and significant problems in psychology. Up to the present time, vast experience in its development from various methodological positions has 
been accumulated, including the principle of a systems approach. However, unlike the situation with some other general psychological problems (for example, such issues as the structural-functional organization of the mental-processes system, the structural organization of personality, the structural level of activities), the systems approach seems to be insufficiently implemented in relation to ability problems. Moreover, in the exploration of this problem, for both historical and epistemological reasons, the tradition of analytical methodology in psychological research - that is, the desire to decompose the whole psyche into its individual components (abilities, properties, the individual qualities of the subject, personality traits) - is very strong. As a result, the current state of the ability problem is characterized by certain peculiarities that, in my opinion, not only allow but strongly require significant intensification of the implementation of the systems approach. And, typically, such peculiarities are found not only when considering particular aspects of the ability problem but also in formulating its most general and fundamental issues.

One of these kinds of issues is the problem of the content of the ability category itself. Moreover, in a sense, this problem is not only major but also fundamental: it is basic for understanding the subject matter of the psychology of abilities. Our interpretation and, on the whole, our understanding of this subject matter depend in many respects on how we solve the problem of the content of the ability category and on how we differentiate the abilities within the general category of abilities.

The solution to this problem, according to the traditional viewpoint, consists in the dichotomous differentiation of the category of abilities into two basic classes or, more precisely, into macroclasses: general and specific (particular) abilities. And although the reasons for such a dichotomous and therefore relatively simplistic possible disjunction concerning such a complex psychic reality as abilities are not quite clear and justified, this categorization has been fixed in the science, has become traditional, if not axiomatic and even "canonical." Inside each of these classes further differentiation and specification of the composition of abilities are possible. Thus, intellect, creativity, learning ability, reflexivity can, for example, be differentiated within the class of general abilities; however, the problem of the composition and, most important, of the criteria for the differentiation of general abilities still remains unsolved. 
The class of specific (particular) abilities seems to be subject to differentiation as well, and here there are two alternative approaches to their differentiation and subsequent study: the personal-operational and the functional-genetic paradigms (Ilyin, 1987). According to the personaloperational paradigm, abilities are classified by the kinds and types of activity; according to the functional-genetic paradigm, abilities are classified in accordance with the basic cognitive mental functions and their corresponding basic cognitive mental processes.

The following significant fact requires special emphasis. All discussions and disputes about and all disagreements and differences in differentiating the general subject matter of the psychology of abilities arise after the above-mentioned dichotomous differentiation of the subject matter into general and specific (particular) abilities has been made. However, the given differentiation itself is not questioned; it is accepted as the original one and, I repeat, as almost axiomatic. Given this situation, the most general and fundamental question - the question of the legitimacy of this differentiation and, most important, its sufficiency for the adequate explication of the total profusion of a person's abilities, of the whole content of the ability category - doesn't arise, and, further, this question cannot arise. As a result, in the psychology of abilities we have a kind of "presumption of nonexistence", according to which it is recognized a priori that there are only two classes of abilities: general abilities and specific (particular) abilities. Thus, the subject matter of the psychology of abilities is (intentionally or not) subject to the original, a priori reduction and, most likely, to impoverishment in comparison with its real content. In this connection, a question arises: Wouldn't it be logical to assume (only to assume) the possibility of the existence of other classes of abilities in the overall structure of the abilities of the personality - the classes that can be reduced neither to general nor to specific (particular) abilities?

In my opinion, in order to try to solve (or, of course, only to reach the first approximation of) this really complex theoretical problem, it is necessary to synthesize the perceptive functional-genetic paradigm for studying abilities (Ilyin, 1987; Shadrikov, 1995) with modern views existing in the theory of mental processes in general and in the concept of the integral mental processes in particular (Karpov, 2000, 2004). And here it is significant that both the functional-genetic paradigm and the concept of the integral mental processes are developed on the basis of 
systems methodology and thus not only are fully "compatible" in the methodological aspect but also serve as a specific "conceptual bridge" between the psychology of abilities (as the subject area of the research) and the systems approach (as the methodology of its cognition).

Indeed, as has already been noted above, from the viewpoint of the functional-genetic paradigm, abilities are differentiated on the basis of their correspondence to the basic cognitive mental functions and their corresponding processes. As a result, sensory, perceptual, imaginative, attentional, mnemonic, and thinking abilities are identified. They form the "core" of specific abilities: they play the most important and even the determining role in the whole system of the abilities of the person. However, in spite of the exceptional and, we can say, unprecedented significance of such a type of abilities, we cannot get away from the simple fact that the cognitive function is only one of the basic mental functions, and cognitive mental processes are only one of the basic classes of mental processes in general. Therefore, to consistently follow the logic of the functional-genetic paradigm, the existence of other abilities corresponding to other basic mental functions (for example, regulatory functions) can't be denied, and, furthermore, their existence is very much possible.

II

In order to implement the assumptions formulated above, it seems expedient to refer to the concept in our literature of the integral mental processes. On the basis of the composition and the structural analysis of the procedural-psychological regulation of activity and behavior, this concept proves the necessity of differentiating the two forms, the two classes (and levels), of the organization of mental processes: (1) the main, traditionally differentiated processes (cognitive, emotional, volitional, motivational) as processes of the "first order"; (2) the synthetic, regulatory processes (goal formation, anticipation, decision making, prognosis, programming, planning, control, self-control) as processes of the "second order."

These processes can be defined as the integral processes of the regulation of activity and behavior. They seem to be a necessary intermediate link, a stage and level of the integration of basic mental processes and an integral regulation structure for activity and behavior. 
Such processes not only exist but also have been known for quite a long time in psychology; they are also reflected in the natural language. However, for them to be adequately differentiated and understood, it is necessary to expand the traditional scheme of the conceptual description of the processes of the psyche, to add the analytical-cognitive set to the study of the regulatory-synthetic set.

The point is that, for the effective and active interaction of the subject with reality, it's not enough to make use of the processes directed mainly to orientation and cognition (cognitive processes); to activation and evaluation (emotional processes); to the stabilization of activity (volitional processes); and to the stimulation, the initiation, of this activity (motivational processes). There is also an objective necessity to involve processes that are aimed specifically at the construction, organization, and regulation of behavior and activities. As such specific regulatory processes we should consider the above-mentioned processes, which are already known but usually not united in a qualitatively specific class of processes designated by us as integral.

In my opinion, it is expedient to consider all psychological peculiarities of the integral processes in the light of the dialectics of the general, the special, and the individual with respect to the characteristics of other processes of the psyche. In other words, these processes have properties in common with all other categories of processes; however, alongside these common properties, they have special, specific characteristics that allow us to consider them a homogeneous (in some significant respects) group of processes. Finally, concerning common and particular characteristics, each of the processes of this group also has its own (singular) characteristics describing its own qualitative peculiarity (originality).

Indeed, generalizing the results of the works devoted to the study of goal formation, anticipation, decision making, planning, prognosis, programming, control, and self-control reveals a number of key features of all these processes that are analogous on the whole to the leading characteristics of other traditionally differentiated processes. So, all of them are psychic in the mechanisms of their implementation; are characterized by the properties of subjectivity, ideality, purposefulness, objectness; have a specific operational structure; are aimed at providing the most common adaptive functions; are complex, multilevel, and systems-organized 
formations; allow implementation in both proper procedural form and in the form of relatively autonomous, expanded activity. The presence of these features, which are shared with other processes, in fact, allows us to assume that all these processes are specific but nevertheless form one of the classes of processes implemented by the psyche.

All these processes, however, possess some features typical of only this class. The main features can be characterized in the following way.

First, the basic criterion for distinguishing all these processes is the correspondence of each of them to a certain complex function according to the organization of activities conditioned by its psychological structure. These functions, which, in their totality, form a closed, integrated circuit of regulation, are the following: goal formation and its differentiation into subgoals, anticipation of the results of an activity (intermediate and final), removal of pragmatic uncertainty (i.e., decision making), formation of an activity program, accomplishment of control and selfcontrol (current and final), and others.

Second, a common feature of this class of processes consists in the fact that all are, by definition, regulatory-monofunctional; in other words, they are aimed at providing a single regulatory function for their organization that is invariant with respect to differences in types and kinds of activities. And in the same way they also differ from other classes of mental processes. Indeed, for example, memory and thinking are necessary for the realization of almost all regulatory functions (they are included in the composition of goal formation, as well as in decision making, self-control, etc.) - that is, they are, in this respect, regulatorypolyfunctional processes. The property of regulatory monofunctionality (the invariance of the function being realized ) is an objective and sufficient prerequisite for making an invariant operational composition of each of these processes.

Third, another basic feature of this group of processes is that they have a comprehensive, synthetic composition; they are integral in their heterogeneity and in the varying quality of the processes of some other categories and classes integrated in them. In addition, any traditional processes included in the composition of the integral ones will always be represented in them only to the extent to which and in the aspect in which they are necessary and sufficient for the realization of the integral process. 
The property of consistency (the system property), which is common in all mental processes, also acquires a definite specific nature in the integral processes. The fact of the matter is that the individual, traditionally differentiated mental processes (cognitive, emotional, volitional, motivational) appear in the quality of their functional components. Here, the composition of the mental processes included in the integral ones seems to remain invariant, but the intensity of each of them when they are included in various integral processes differs significantly. The structure of the functional links among them, established in a variety of integral processes, also varies. The integral processes being formed represent a variety of psychological systems. The ratio of "primary" and "secondary" - that is, of the integral mental processes, - acts as a ratio of a component and the system. The specific content of the system consists of the effects of mental integration and the generative phenomena and mechanisms associated with them.

Fourth, a general characteristic of the integral processes is, then, that they all have a common and initial specifically regulatory direction; they are characterized by the singularity of their functional purpose as compared with other groups of mental processes. So, for example, cognitive mental processes realize both cognitive (mainly) and regulatory functions; emotional processes, mainly evaluative and activational functions. But the integral processes are aimed primarily and directly at the implementation of regulatory functions proper. In other words, these processes, together with the regulatory potential of all other mental processes, are a part of the regulatory subsystem of the psyche and form its specific content. From this viewpoint, there appears to be a need to differentiate cognitive and regulatory processes.

Activity as an objectively existing form implies the same objective character of the processes of its organization - integral psychic processes. Together they constitute the transitional level of the integration between individual mental processes and the comprehensive regulation of activity and behavior. As such, the totality of integral processes is a most important aspect, revealing the actual procedural content of the overall structure of the self-regulation of activity.

Fifth, another feature of the integral processes is the fact that their operational composition and contents are not limited to the composition and contents of the additive sum total of those "primary" mental 
processes that implement them. The point is that they are always under construction and are organized according to the type of targeted action (in advanced forms, activity). This organization is recorded in the synonymy of expressions of the type "the process of control" and "the action of control," "the decision-making process" and "the actions of decision making." In other words, the operational structure of these processes is determined by the structural and functional organization of the action. Therefore, they are at the same time both processes and actions, and their adequate and full description implies the use of the principle of complementarity in understanding them as both processes and actions.

Sixth, a distinctive feature of this class of processes is that they act as multi-integrative; in other words, the manifestations of integrative mechanisms can be found in them from a number of different directions: (1) from the complexity and synthesis of their procedural content and composition; (2) from the integrative nature of the main mechanisms of their organization; (3) from their general functional purpose, their orientation to the organization, in essence, of the integrated activities; (4) from the complexity and synthesis of their operational and component composition; (5) from their genetic aspect: their formation and development in ontogenesis are at the same time an increase in the degree of integration of the psyche in general. Through them and "in them" the developing psyche increases the extent of its integrity and integration and is formed as the "absolute whole," as a "fully interrelated system."

Seventh, a specific feature of the integral processes, which is expressed, however, only their integral structure (but is not typical of each of them separately), is the following pattern of their organization. Among them one cannot isolate a single process that is consistently located at the top of the hierarchy of the regulatory subsystem. Any of the integral processes, depending on the specific situation, may become the leading and highest and may arrange for the purposes of its implementation all other integral processes. It is also significant that the change of the integral processes on the main level occurs quite naturally and depends on the current situation in regard to the content and the conditions of the specific operational and behavioral problem. 
All these features can be thought to indicate that the organization of the integral processes obeys not a hierarchical but a heterarchical principle. As is well known, such an organization is characterized by flexible and dynamic redistribution, a change of governing centers caused by a specific situation; it also has simultaneously several equal governing centers.

Thus, through the formation of the system of the integral processes the psyche enriches the arsenal of the functional principles of its organization. The principle of hierarchy (on which, in particular, the cognitive subsystem is based) is supplemented by the principle of heterarchy: the organization of the regulatory subsystem is based on it. The synthesis of these two most common and powerful principles - hierarchical and heterarchical - is the basis of the highest capacity for organization and coordination in the effective management and self-control of the psyche.

One of the most important features of these processes, which is attributively inherent in them, is their initial regulatory direction (which provides one or another of the basic functions of the organization of activities and behavior). Moreover, this direction is invariant in relation to the different forms, types, kinds, and even psychological statuses of activities. The psychological status of activities is the differentiation of the "external" and the "internal" - that is, proper psychic activity, "the mental as an activity." In this activity, all the integrative, regulatory processes act as a system of self-regulation, forming the procedural "core" of reflection as such.

Indeed, in relation to the "internal" activity - the ideal, proper mental activity - as well as in relation to the externally transforming activity, the means of its organization and regulation are required with the same objective necessity. However, because the internal activity in its fundamental features is isomorphic to the external one, the tools originally developed for the implementation of external activity can and should be used as such means. This is again nothing other than the system of the integral processes. The class of the integral processes starts "serving" the inner activity, is transposed into it.

A regulatory invariant, which is reflected in the content of the class of the integral processes and which initially serves the external activity, 
starts to regulate the internal activity ${ }^{1}$. Thus the regulatory invariant becomes the self-regulatory invariant.

Here, the rather peculiar phenomenon of "activity reflectiveness" takes place. It consists in the fact that, in relation to internal, proper mental activity, operational tools that originally have been formed in the activity and therefore have the same nature start to be used as its main regulators. This is the system of the integral processes of regulation. Thus the architectonics, the structure and the principles of the activity, "turn around" on themselves; the phenomenon (and mechanism) of activity reflectiveness takes place. This phenomenon is therefore a real basis for the reflective regulation of the activity, as well as for reflection as a process in general.

Activity reflectiveness is manifested most clearly in the most complicated forms of internal activity - for example, in intellectual activity, in the intellect as such. In relation to it, or rather to its dynamic aspect, the system of the integral processes (goal formation, anticipation, decision making, prognosis, planning, programming, control, self-control) acts as a system of cognitive and regulatory meta-operations (metameans), as a system of metacognitive regulation. It coordinates and organizes the work of all the other intellectual functions and mechanisms.

My research shows that the measure of a subject's development of the integral-processes system significantly correlates with general intel-

${ }^{1}$ I define the concept of regulatory invariant as a system of processes that is necessary and sufficient for generating, organizing, and regulating some overall behavioral (activity) act and that constitutes a closed "regulatory ring." It therefore includes those processes that are part of the class of integral ones, but with two additions. First, a regulatory invariant gives those processes a chronological order. The temporal order of a regulatory invariant is as follows: forming goals $\rightarrow$ anticipating future results $\rightarrow$ making decisions regarding the methods and means of an activity $\rightarrow$ making prognoses of possible changes in the conditions of an activity, in one's own actions, and in their results $\rightarrow$ planning an activity (including a number of possible options for its deployment) $\rightarrow$ programming an activity (already assuming the choice of this or that option, its time organization) $\rightarrow$ the performance itself $\rightarrow$ controlling the (current and final) performance $\rightarrow$ exerting self-control (also current and projected) $\rightarrow$ making corrections by comparing the achieved results with an ideal goal and the anticipated results. Second, a regulatory invariant has a closed character: results of the control are correlated with the initial ideas about the goals of the activity (as an aspect of the goal-formation process) through feedback, and this process may result in a correction of one of two types, either compensatory or transforming. 
ligence level (Karpov, 2000). Therefore, on the basis of the metacognitive regulation of the intelligence forming one of its highest levels, there also is a system of specifically regulatory-integral processes.

To conclude this discussion of the general characteristics of the class of integral mental processes, it is also necessary to point to the fact that, as follows from the results received (Karpov, 2000, 2004), each of these processes can provide some regulatory function with a varying degree of efficiency. In simpler words, any of them (as well as other mental processes) has an individual measure of intensity.

\section{III}

As shown by the analysis in the previous section, in the system of the integral processes there are processes that (1) are orthogonal with respect to all other procedural entities of the psyche (although, of course, they are closely interrelated with these entities and, further, are derived from them); (2) are differentiated according to the criterion of objective correspondence to some regulatory function; (3) have an individual measure of intensity, independent of the type and kind of activity implemented by the subject. On the basis of this analysis we may assume that in the system of the integral processes there exists a category of abilities - the integral abilities of a person - that are as specific as the processes themselves. They are orthogonal with respect to other categories of abilities to the same extent that the integral processes have the same property of orthogonality with respect to other classes of mental processes. For this reason there are grounds for placing this category of abilities in a specific class. This conclusion fully corresponds to the modern, functional paradigm of the psychology of abilities, as the abilities are differentiated solely on the basis of the category of "the function" (Karpov, 2004). To tell the truth, they are specifically regulatory functions; but it is precisely this characteristic that gives them qualitative originality, makes us speak of them as of the regulatory abilities of a person. Having an individual measure of intensity, they are invariant in relation to the kinds and types of activity. Consequently, they are characterized by subject conditioning and act as abilities.

Thus, the entire set of methodological and theoretical, experimental and empirical data allows us to put forward an assumption of the existence of a specific category of abilities - the integral abilities of the 
individual ${ }^{2}$. The main argument in favor of this assumption is the direct correspondence of these abilities to the integral processes of the mental regulation of activity and behavior (both in general and in each individual component for each of the processes). But, inasmuch as the orthogonality of this class of processes, the impossibility of reducing it to any other class of mental processes, has been proved earlier, the same conclusion must, apparently, be drawn with respect to the category of the integral abilities as well. Similarly just as ideas about the integral processes contributed to the development of ideas about the procedural content of the psyche, the study of the integral abilities may contribute to the clarification and deepening of views on the composition and structure of the subject's abilities on the whole. And, above all, this clarification of views can result from clarifying the nature of the psychological status of these abilities.

Indeed, as has already been noted, at present the traditional and generally accepted differentiation of abilities is the differentiation into two main categories: specific (particular) and general. The specific category can also be differentiated into two forms depending on the accepted common approach: either personal-operational (person-activity) or functional-genetic (Ilyin, 1987; Shadrikov, 1995). In accordance with the personal-operational paradigm, abilities are subdivided into kinds and types of activity, and, in accordance with the functional-genetic paradigm, they are subdivided into basic mental functions and the basic mental processes related to them.

In light of this differentiation it is quite obvious that the integral abilities in principle cannot be identified with either specific or general abilities; they do not "fit" into either of the two traditionally differentiated categories. Indeed, if we accept the functional-genetic paradigm of specific abilities (in my opinion, it is the more adequate), then none of the integral abilities, by definition, can be localized at the level of specific abilities, as it forms as a product of the integration of the entire system of cognitive functions and cognitive processes (and not only cognitive but also other

2 To avoid confusion it is necessary to make the following terminological clarification. Strictly speaking, almost any ability, including the simplest of them, is integral, synthetic, provided with a whole set of means and mechanisms. In this context, however, the issue is different: a certain class of abilities directly correlates to a certain class of processes - to the integral mental processes - and thus finds its direct terminological designation. 
kinds of functions and processes). If we accept the personal-operational (person-activity) paradigm, neither of the integral abilities also can be regarded as specific - that is, corresponding to this or that particular activity - as they all, as shown above, have a kind of "over-the-activity" nature and manifest themselves invariantly in different kinds, types, and even forms of activities ("external" and "internal").

In addition, all integral abilities cannot be localized in the system of general abilities of the individual either. Being integrative in one crucial respect (that is, acting as a product of the synthesis of other, local, abilities and procedural entities), these abilities at the same time are differentiated in another, no less important, respect. The fact is that all of them are the result, the product, of the differentiation of the mental regulatory subsystem and therefore cannot possess the status of the maximum generalization. They therefore are identified not on the extremely generalized level of mental functioning but on the level of its basic subsystems (such as the cognitive, regulatory, and communicative subsystems), and they differentiate the regulatory subsystem. In addition, they have one more feature that distinguishes them from the general abilities: general abilities are characterized by relatively higher "cognitive specificity," but integral ability is a specific regulatory ability.

Therefore, the specific nature and status of the integral abilities is that they are located between the levels of specific (particular) and general abilities; thus they form the mesolevel in the structure of the abilities of the personality. The mesolevel is located in the continuum between the macrolevel of the general abilities of the individual and the microlevel of the specific abilities. And, in this regard, it is logical to make one more assumption, also a general plan.

In my opinion, it is more helpful to interpret the traditional differentiation of abilities into general and specific not taxonomically, as a division into groups or categories, but continually, as two poles on a continuum of the definiteness of abilities. They are not so much two categories differentiated in only one possible way but rather the poles of a continuum of abilities that are most significantly different from each other. And here a general gnoseological rule works: the poles of a continuum of some quality (definiteness) are recognized, as a rule, easier and earlier than is the middle of the continuum (that is, the intermediate values on the continuum) (Vekker, 1974). So psychological knowledge first recognizes the 
specific and general abilities as the poles of the ability continuum. Next, however, is the question of the possible existence of abilities within this continuum (and this is just the question I answer in this article by means of formulating a hypothesis about the integral abilities). However, this understanding of the composition of abilities is, strictly speaking, only a step on the way to their cognition and an adequate explication of their actual structure.

The fact is that the continuum of abilities is not one-dimensional, but it is multidimensional. It is a "ladder" of definiteness; that is, it is the manifestation of the layered structure of the organization of abilities. Thus the assumption is that the total structure of the abilities of an individual is built up on the basis of levels. At one pole ("the bottom") the microlevel of specific abilities is located; at the other ("the top") is the macrolevel of general abilities. A mesolevel consisting of the integral abilities is located between them.

In connection with this conclusion two rather important theoretical problems can be formulated. First, does the layered structure of abilities include only these three levels? In principle, this possibility is not excluded, but the existence of some other levels and categories of abilities that are not covered by these three levels is also possible. It would be presumptuous to believe that the three, already-known, levels cover the entire hierarchy. Second, there is the issue of how the mechanisms and regularities of the ability level interact within the overall hierarchy. How are the general and specific abilities related? Are the integral abilities synthesized from the specific ones and the general ones on the basis of the integral? What are the principles for structuring the levels in the general hierarchy of the abilities of the personality? What does the hierarchical organization of abilities provide in regard to general adaptation? These and many other theoretical issues arise in the transition from the traditional (taxonomic) to the continual and, further, to the structural level of abilities.

In general, it seems somewhat strange that ideas of the structurallevel approach, widely and reasonably implemented in relation to many of the most important psychological problems (the problem of mental processes and states, the structure of personality, the structure of activity, and so on), for the time being are not sufficiently assimilated by the psychology of abilities. But the implementation of such an approach can 
contribute to the solution of major problems of the psychological theory of abilities.

So, in particular, the differentiation of the mesolevel of the integral abilities, in fact, reduces the sharpness of the counterposition of the two main paradigms of ability theory - the personal-operational and the functional-genetic. Indeed, the integral abilities are attributively related to the category of regulatory processes, and those, in turn, are differentiated according to the criterion of their correspondence with this or that basic function of the organization of activities or behavior.

Thus, the integral abilities are also differentiated according to this functional criterion. Therefore, the integral abilities, without losing their regulatory status, allow their "functional" explication. Moreover, any of the basic functions of the organization of activities (again, by definition) requires synthesis - the integration of all proper mental functions, spread out on the basis of their processes (perceptual, mnemonic, thinking, and others). Therefore, each integral ability, assuming all mental functions and "consisting" of them, is not reduced to them (or rather, to their additive totality). The way these mental functions (and their corresponding local abilities) are synthesized is determined by the content of other functions - regulatory functions, those related to the organization of the entire activity. It follows that the integral abilities are at the same time both a system of mental functions and activity entities. Their adequate and full disclosure is possible on the basis of the principle of their complementarity both as the properties of functional systems (mental functions) and as the components of activity (regulatory functions). Therefore, both the ontology of the subject and the morphology of the activities are synthesized in them. The ontology of the subject takes the form of a hierarchy of abilities; the morphology of the activities is in the form of the structure of the regulatory functions.

It is necessary to emphasize another point. The system of integrative, regulatory processes of the organization of an activity is nothing other than the procedural content of some regulatory invariant - that is, the totality of the processes that are necessary and sufficient for the regulation of the activity as such and do not depend on its specific modifications (Karpov, 2004; Karpov \& Skityaeva, 2005). In fact, in psychology, generally speaking, the activity and the system of integral processes of its regulation are in many respects synonymous, in many respects identical 
in content. As has been shown in a number of works (Karpov, 2004; Karpov \& Skityaeva, 2005), the very development of an activity as a specific form in ontogenesis has as one of its main mechanisms the formation of the integrative processes.

I believe that the system of the integral processes is the "key," the missing link, which is necessary for explaining the phenomena and mechanisms for generating internal, proper mental activity on the basis of external activity. The psyche is "born" from the activities or the subject activity not on the basis of an abstract and not quite definite mechanism for interiorization but by mediating the creation, development, and improvement of the system of integral processes. Originally organized in accordance with "the logic of reflection," the psyche starts therefore to gradually reproduce in itself and, in general, to be developed according to the "logic of activities." It thus significantly expands its potential and operational opportunities, develops as an internal activity, and acquires in this form self-motion and self-development. Therefore, the very concept of the internal activity acquires in this form not only a metaphorical and indirect sense but also a direct, immediate meaning. Its procedural-psychological content transposes to itself the architectonics of the external activity (its structure) and develops as the activity, although already in a different, a perfect, way.

In light of the above discussion, it is logical to assume that the formation of the integral processes has as its basis certain prerequisites in the form of corresponding abilities - the regulatory abilities. Therefore, through the formation and development of the regulatory processes of the organization of activities, those abilities that are isomorphic to them - integral, regulatory - also develop. Because the range of the development of the activity system is very large (actually from "zero" up to higher forms of the organization of activities), the range of the genesis of the integral abilities is also large. Hence, we can assume that the integral abilities are characterized by the most pronounced genetic dynamics, that they are the most labile component in the hierarchical structure of abilities. Being activity-specific, they are the most sensitive in relation to the development of activities as a whole and the basic functions of this development in particular. In addition, being one of the levels of the overall ability structure (the mesolevel), they, by definition, are related to its other levels, the levels of the general and specific abilities. 
The level of interaction is reflected in the genetic aspect as well. It is therefore possible that one of the major mechanisms of the genesis of abilities, of their whole structure (including both specific and general), is the development of the integral abilities of the person.

Finally, it is necessary to note one more consequence of the views developed above. The system of the integral, proper regulatory processes can realize their functions not only in relation to the "external" but also in relation to the "internal" - that is, proper mental activity. In this case, as noted above, it can be seen as a system of self-regulatory processes, and it forms the procedural content of conscious, arbitrary regulation. The synthesis of these self-regulatory processes has an adequate subject representation and phenomenologically appears as a reflexive regulation. Thus each of the integral processes is revealed in the function of one of the operational components of an even higher-order process - the meta-integral reflection process.

The approach formulated above allows us to specify the notion of the structure of reflexive (i.e., metacognitive) regulation, the structure of reflection as a process. Revealing the initially activity-based regulatory nature of this process, the approach formulated shows that the set of all basic integral processes may be included, when necessary, in the composition of reflection. However, these processes are included in the composition of reflection because of their focus not only on the solution of directly regulatory tasks but also on the solution of self-regulatory problems - that is, in relation to the organization of internal activities. Therefore, reflection as a process synthesizes the entire system of integral processes and to a large extent is involved in such a synthesis. Those properties that are usually identified by means of analysis in the process of conscious regulation (broadly, in consciousness as in the phenomenon) are the properties of each integral process and their sum total: purposefulness, anticipation of the results of behavior (anticipating), freedom of choice (decision making), establishment of orderliness and meaningfulness in the behavior (planning and prognosis), "being aware of one's actions" (control and self-control), and so forth. The presence of such properties in the processes included in reflection determines the phenomenology of consciousness as such and lies in the basis of reflection not only as a process but also as a state.

In addition, because reflection as a process is derived from the synthesis of its operational components (integral processes, as well as 
other, "secondary," primarily metacognitive, processes), the individual measure of its intensity (reflexivity as an ability) has an even higher status, extending beyond the limits of the mesolevel of the organization of abilities. Therefore, this conclusion confirms my previously developed position, according to which reflexivity should be interpreted as a general ability (alongside the other general abilities - intelligence, creativity, ability to learn) (Karpov, 2004). Thus, the category of the integral abilities not only contributes to the development of ideas about their holistic, structural-level organization but also allows us fully and with discrimination to reveal the composition of the general abilities of the individual as a higher level in this organization.

\section{References}

Ilyin, E.P. (1987). Problema sposobnostej: dva podhoda ee reshenija [The problem of abilities: Two approaches to solving it]. Psihologicheskij zhurnal [Psychological Journal], 2, 23-31.

Karpov, A.V. (2000). Obshchaja psihologija subektivnogo vybora [General psychology of subjective choice]. Moscow: Institut psihologii RAN.

Karpov, A.V. (2004). Metasistemnaja organizacija urovnevyh struktur psihiki [Metasystem organization of the level of psychic structures]. Moscow: Institut psihologii RAN.

Karpov, A.V., \& Skityaeva, I.M. (2005). Psihologija metakognitivnyh processov lichnosti [Psychology of metacognitive processes of the personality]. Moscow: Institut psihologii RAN.

Shadrikov, V.D. (1995). Dejatel'nost' i sposobnosti [Abilities and activities]. Moscow: Meditsina.

Vekker, L.M. (1974). Psihicheskie processy [Mental processes], vol. 1. Leningrad: Leningrad State University. 